Supplement Issue 1: International Tehran Breast Cancer Congress (TBCC9)

\title{
Checking Herbal Products of Resveratrol on Preventing Breast Cancer and Increasing Cell's Apoptosis
}

\author{
Mohamadali Mirjalili ${ }^{1,}$, Amirreza Rafiei ${ }^{1}$, Amirmohamad Naghibi ${ }^{1}$ \\ ${ }^{1}$ Shahidsadooghi University, Yazd, Iran \\ ${ }^{*}$ Corresponding Author: Mohamadali Mirjalili, Shahidsadooghi University, E-mail: \\ hoodream1376@gmail.com
}

DOI: $10.21859 /$ mci-supp-61

\section{Keywords:}

Herbal

Resveratrol

Breast Cancer

Cell's Apoptosis

\begin{abstract}
Introduction: Breast cancer is the most common cancer and it is the major reason for death of women's in the world. Breast cancer is first in four of all female cancers in Iran. It seems herbal products have fewer side effect in curing breast cancer compared with other methods of preventation. Resveratrol is a member of a compounds called polyphenols which are found in herbal products. This study reviews resveratrol effect in preventing breast cancer and increasing cell's apoptosis.
\end{abstract}

Materials and Methods: This article is a systematic review which has been searched in google scholar and pubmed data base with key words of "resveratrol, breast cancer, apoptosis" until the half of 2016 and then found articles were checked and reviewed.

Results: Resveratrol is a polyphenol with the antioxidant properties and with 3.4.5 three hydroxy trans stealen formula which is in many herbal products including grapse, raspberry, berry, peanut, blueberries, etc. Resveratrol lead to stop the proliferation of various human tumor lines in vitro's conditions. Anti-tomors activities of resveratrol is intermediated by several cellural cycles, preventing from proliferation of tumors, inducting apoptosis, decreasing inflammation, and vascularization and preventing from spreading and metastasis and because of height anti-tumor and anti-estrogenic actions it can be a good substituation for hormone therapy and other ways of preventing breast cancer.

Conclusions: Resveratrol in cellular and molecular levels with effects on gene expression can lead to apoptosis and with stimulating lipolysis and inhibiting lipogenesis, can prevent obesity and decrease fat mass which one of the most important risk factors in breast cancer. 